\title{
Low-Voltage Electron Microscopy (LVEM) of Carbon Nanotubes and Nanoporous Poly(3,4-ethylene dioxythiophene) (PEDOT)
}

\author{
Jinghang $\mathrm{Wu} *$ Bong Sup Shim ${ }^{* *}$ and David C Martin ${ }^{* * *}$
}

*Macromolecular Science and Engineering, University of Michigan, Ann Arbor, MI 48109;

Materials Science and Engineering, University of Delaware, Newark, DE 19716

**Materials Science and Engineering, University of Delaware, Newark, DE 19716

*** Materials Science and Engineering, University of Delaware, Newark, DE 19716; Materials

Science and Engineering, Macromolecular Science and Engineering and Biomedical Engineering University of Michigan, Ann Arbor, MI 48109

The low-voltage electron microscope (LVEM) is a table-sized electron microscope developed by Delong Instruments. The LVEM can operate in transmission electron microscopy (TEM), scanning transmission electron microscopy (STEM), scanning electron microscopy (SEM), and electron diffraction (ED) modes. The LVEM's capability of operating in different modes on the same sample provides powerful complementary information about the materials under study. It has a $5 \mathrm{kV}$ electron source that is a much lower voltage than conventional electron microscopes that range from $60 \mathrm{kV}$ to $400 \mathrm{kV}$. This enhances the image contrast of low atomic number samples dramatically by increasing electron scattering. This LVEM opens up the possibility of avoiding the heavy metal stains typically used in imaging polymeric and organic molecular materials while preserving reasonably high resolution $(<2.5 \mathrm{~nm})^{1}$. In this study, we demonstrate the high contrast LVEM imaging of low atomic number materials including single strands of single-wall carbon nanotubes (SWCN) ( $\sim 1 \mathrm{~nm}$ diameter) as well as multi-wall carbon nanotubes (MWCN). We also have investigated the morphology of nanoporous poly(3,4-ethylenedioxythiophene) (PEDOT) thin films. PEDOT is a conducting polymer of interest for interfacing electronic biomedical devices with living tissue.

The samples of SWCN and MWCN were dispersed into sodium poly(styrene sulfonate) aqueous solutions and then nebulized onto copper grids with nanometer thick amorphous carbon film. The LVEM readily images the SWCN and MWCN samples with high contrast.

PEDOT samples were made by oxidative polymerization in a bicontinuous non-ionic surfactant cubic phase. Drops of the nanoporous PEDOT suspension in ethanol were deposited onto copper grids coated with $\sim 2.5 \mathrm{~nm}$ thin carbon films. The stained samples were exposed to osmium tetroxide vapors for an hour. The nanosized pores were disrupted during the osmium staining process, while the unstained nanoporoous PEDOT thin films were hardly visible at all when using a JEOL TEM with a $200 \mathrm{kV} \mathrm{LaB}_{6}$ gun. This demonstrates the benefits of using LVEM over normal TEM for studying the original morphology of unstained polymeric materials at high resolution. 


\section{References}

[1] L.F. Drummy et al., Ultramicroscopy. 99 (2004) 247.

[2] This research was supported in part by the National Science Foundation (NSF) and the ACS Petroleum Research Fund (PRF).

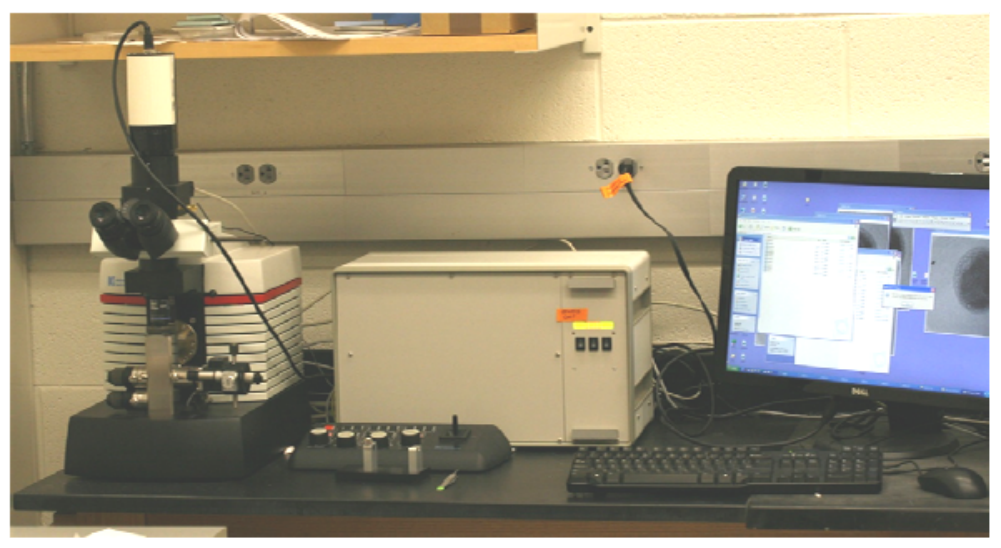

FIG. 1. Picture of table sized LVEM

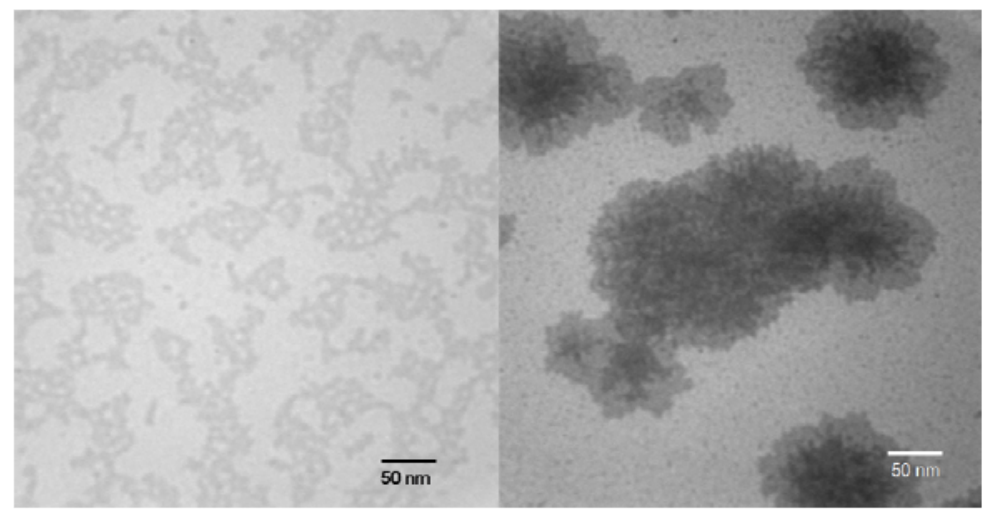

FIG. 2. LVEM micrographs of PEDOT cubic phases (left: unstained, right: stained with osmium teroxide)

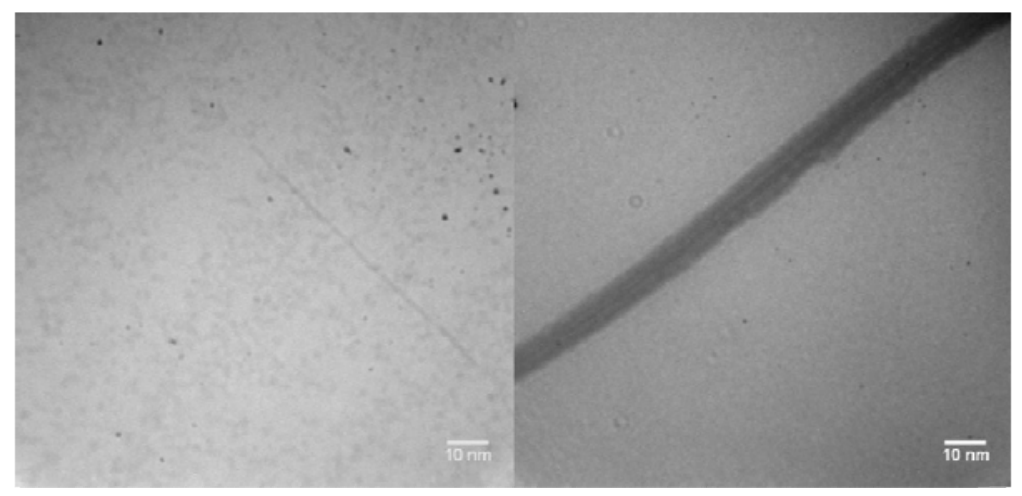

FIG.3. LVEM micrographs of Carbon nanotubes (left: single wall, right: multiwall) 